\title{
Serological and Finger-printing Analyses of Mutant Flagellar Antigens of Salmonella
}

\author{
By S. YAMAGUCHI AND T. IINO \\ National Institute of Genetics, Yata I,III Misima, Sizuoka-ken, Japan
}

(Accepted for publication 23 October 1970)

\begin{abstract}
SUMMAR Y
Spontaneous mutants with altered flagellar antigen were isolated from a phase-I stable Salmonella strain, TR6, whose phase-I antigen consists of at least three antigenic factors, $\mathrm{g}_{3}, \mathrm{~g}_{4}$ and $\mathrm{f}$. Three kinds of antisera reacting with each of these factors were used as the selective agents. The mutants were isolated by picking from swarms in semisolid medium containing the respective antisera. Mutants were classified into two groups. A group comprising four mutants selected with antiserum to $\mathrm{g}_{3}$ and two mutants selected with antiserum to $g_{4}$, in which all three antigenic factors examined were simultaneously altered; and a second group comprising all the other mutants, in which only a single factor corresponding to the selective antiserum was altered. All the altered factors retained weak cross-reactivity with the original factors. In all cross-absorption-agglutination tests between each of the mutant antigens and the wild-type antigen, activity of the antisera was fully absorbed, showing that the mutant antigens had neither lost any antigenic specificities nor gained any new specificity. Transductional analysis showed that the sites of mutation were inseparable from $H I$, the structural gene for phase-I flagellin. Tryptic peptide patterns of flagellins of two mutants were compared with that of the wild-type strain and a difference of one or two peptides was detected. From these results it is concluded that these mutants possessed a minor change in primary structure of flagellin which resulted from a mutation in the $\mathrm{HI}$ gene.
\end{abstract}

\section{INTRODUCTION}

Salmonella flagella are composed of a single kind of protein, called flagellin, of molecular weight about 40,000 (Kerridge, Horne \& Glauert, 1962; Asakura, Eguchi \& Iino, 1964; McDonough, 1965). A molecule of flagellin is regarded as the unit of flagellar antigen (Iino, I969). The genes $H_{I}$ and $H_{2}$ in Salmonella which were originally defined as the genes determining the antigenic specificities of phase- $I$ and phase-2 flagellar antigens, respectively, are now known as the structural genes for flagellin in respective phases (Iino, 1969). Therefore, the fine structure analysis of the $H$ genes combined with the serological analysis of flagellin is expected to provide valuable information for an understanding of the genetic determination of the antigenic character of a protein.

Recombination analyses carried out between the antigenic specificity-determining sections which are assumed to correspond to the respective antigenic determinants on phase-I flagellin molecules demonstrated that they are arranged linearly within $H_{I}$ gene (Yamaguchi \& lino, 1969).

In addition to intragenic recombination analyses, the comparative study of mutants 
with altered flagellar antigen serves as an approach to understanding the genetic control of the antigenicity of flagellar protein. Motile Salmonella bacteria placed in an appropriate semisolid medium migrate rapidly through the medium, but the addition of a small amount of antiserum specific to the flagella of the organisms effectively immobilizes them and confines their growth to the site of inoculation. However, antigen mutants whose motilities are not inhibited by the antiserum are often detected because they spread through the medium from the site of inoculation and form swarms. Joys \& Stocker (1966) isolated eight mutants with altered forms of antigen i from Salmonella typhimurium in semisolid medium containing anti-i serum. They showed that the mutants of antigen $i$ had lost some of the antigenic specificities of the wild-type antigen, while they had some specificities absent from it, and that in all these mutants the sites of mutation were in $H I$. In the flagellins of four of these mutants a single difference from the wild-type flagellin was detected in the tryptic or peptic peptide maps by McDonough (1962). Attempts to infer the order of mutations within $H_{I}$ by three-point crosses were unsuccessful (Joys \& Stocker, I969).

The present paper deals with the serological and finger-printing analyses of the mutant forms of a g-complex flagellar antigen, fg. Anti-factor(s) sera were used as selective agents for the isolation of the mutants.

\section{METHODS}

Bacteria. Salmonella strain TR I6 was used for the isolation of antigen mutants, and strains TR6, TR II, TR I6 and TR I7 used for the preparation of anti-factor(s) sera. These strains are the derivatives of a phase-I monophasic strain of Salmonella abortusequi, strain SJ 24I, in which phase-I antigen a of SJ 24I had been replaced by different g-complex antigens following transduction of $H I-g . \ldots$ alleles from the naturally occurring g-complex antigenic Salmonella strains. All of them are phase-I monophasic. The factor compositions of their phase-I antigens (Yamaguchi \& Iino, 1969) as well as antigen types according to the Kauffmann-White scheme (Kauffman, I964) are listed in Table $\mathrm{I}$.

Table I. Salmonella strains used: derivatives of Salmonella abortus-equi SJ24I, with $H \mathrm{I}-\mathrm{g}$. . . alleles transduced from $\mathrm{g}$-complex antigenic Salmonella

All the strains are stable in phase-I.

\begin{tabular}{|c|c|c|c|c|}
\hline \multirow{2}{*}{ Strain no. } & \multirow[b]{2}{*}{ O-antigen } & \multicolumn{2}{|c|}{ Phase-I antigen } & \multirow[b]{2}{*}{ Donor of $H I-g . \ldots$} \\
\hline & & Antigen type* & Factor composition $\dagger$ & \\
\hline & 4,12 & gt & $\left(\mathrm{g}_{0}\right), \mathrm{g}_{1}, \mathrm{~g}_{2}, \mathrm{~g}_{3}, \mathrm{t}$ & Salmonella sp. su 6 \\
\hline TR I I & 4,12 & gp & $\left(\mathrm{g}_{0}\right), \mathrm{g}_{1}, \mathrm{~g}_{4}, \mathrm{~g}_{5}, \mathrm{p}$ & S. dublin NCTC 4197 \\
\hline TR I6 & 4,12 & fg & $\left(\mathrm{g}_{0}\right), \mathrm{g}_{3}, \mathrm{~g}_{4}, \mathrm{f}$ & S. derby NCTC I 729 \\
\hline TR I 7 & 4,12 & $\mathrm{gm}$ & $\left(\mathbf{g}_{0}\right), \mathbf{g}_{1}, \mathbf{g}_{2}, \mathbf{g}_{4}, \mathbf{g}_{5}, \mathrm{~m}$ & S. enteritidis NCTC 4196 \\
\hline
\end{tabular}

* According to the Kauffmann-White scheme.

$\dagger$ Details of the determination of the factor composition have been described in Yamaguchi \& Iino (1969).

Media. Nutrient broth was composed of I $\%(w / v)$ peptone and I $\%(w / v)$ meat extract, adjusted to $\mathrm{pH} 7 \cdot 2$. Nutrient agar was prepared by the addition of $\mathrm{I} \cdot 5 \%(\mathrm{w} / \mathrm{v})$ agar to the mutrient broth. Semisolid medium was prepared by adding $0.35 \%(\mathrm{w} / \mathrm{v})$ agar and $8 \%(\mathrm{w} / \mathrm{v})$ gelatin to the nutrient broth. All the cultures were incubated at 
$37^{\circ}$. When necessary, antiserum was added to the semisolid medium at an appropriate concentration.

Serological procedure. Actively motile bacteria after incubation overnight were suspended in $0.5 \%$ formalin-containing saline. Rabbits were immunized with a total of $6 \mathrm{ml}$. of the bacterial suspension (about $10^{8}$ organisms $/ \mathrm{ml}$.) by one intraperitoneal and five successive intravenous injections at 3 day intervals, and bled $\mathrm{I}$ week after the final injection. Antibodies specific to the somatic (o) antigens were removed by absorbing the serum with bacteria of a non-flagellate mutant of SJ $24 \mathrm{I}$.

Absorption of antisera was done as follows. Antiserum to be absorbed was mixed with absorbing organisms in the proportion of $\mathrm{I} \cdot 0 \mathrm{ml}$. serum to $0.5 \mathrm{~g}$. wet weight organisms. After incubation at $37^{\circ}$ for $2 \mathrm{~h}$., the mixture was centrifuged at 3000 rev./ $\mathrm{min}$. for $30 \mathrm{~min}$. This process was repeated until absorption finished.

Three kinds of anti-factor(s) sera, anti- $g_{3}, t$, anti- $g_{4}, g_{5}, p$ and anti-f, for the selection of antigen mutants were prepared by the above absorption procedure. The original antisera and the absorbing organisms are listed in Table 2.

Table 2. Preparation of anti-factor(s) sera

\begin{tabular}{|c|c|c|}
\hline $\begin{array}{l}\text { Anti-factor(s) serum } \\
\text { to be prepared }\end{array}$ & Original serum & Absorbed with \\
\hline Anti-g ${ }_{3}, t$ & Anti-TR 6 & TR I7 \\
\hline Anti-g $g_{4}, g_{5}, p$ & Anti-TR I I & TR I 6 \\
\hline Anti-f & Anti-TR I 6 & TR 6 and TR I I \\
\hline
\end{tabular}

The titres of antisera were determined by tube agglutination and expressed as the reciprocal of the highest dilution showing activity.

Transduction of $\mathrm{HI}_{\mathrm{I}}$ gene. Phage $\mathrm{P}_{22}$ was used as transducing phage. The phage was propagated on a donor strain by the soft-agar-layer method and the lysate mixed with an overnight broth culture (about $10^{9}$ organisms $/ \mathrm{ml}$.) of the recipient strain at a multiplicity of about 5. After $10 \mathrm{~min}$. at $37^{\circ}$ the mixture was diluted $10^{-1}$ and $10^{-2}$ with nutrient broth, and the dilution brushed in line on semisolid medium containing just sufficient antiserum specific to the flagellar antigen of the recipient for immobilization. After incubation for $15 \mathrm{~h} ., H_{I}$ transductants which appeared as swarms were isolated and their flagellar antigen examined by slide agglutination or by observing their spreading ability in semisolid media containing anti-factor(s) sera.

Preparation of flagellins and their tryptic peptide maps. Procedures for preparation and tryptic digestion of flagellins were as described in our previous paper (Yamaguchi \& Iino, I969). A digested sample was applied to a sheet $(40 \mathrm{~cm} . \times 30 \mathrm{~cm}$. $)$ of Toyo no. 50 filter paper and subjected to electrophoresis in pyridine + acetic acid + water (I35+ I5+ I by vol.) at $\mathrm{pH} 6.65$ for $4 \mathrm{~h}$. at $\mathrm{I} 5 \mathrm{~V} / \mathrm{cm}$. After drying, the paper was subjected to descending chromatography with $n$-butyl alcohol + acetic acid + water $(20+3+7$ by vol.) and the peptide spots developed with ninhydrin.

\section{RESULTS}

\section{Isolation of serological mutants}

Flagellar antigen $\mathrm{fg}$ of TR $\mathrm{I} 6$ has at least three factors, $\mathrm{g}_{3}, \mathrm{~g}_{4}$ and $\mathrm{f}$, as shown in Table $\mathrm{I}$. The term 'antigenic factor' means the antigenic unit detected by means of the crossabsorption-agglutination test. Three anti-factor(s), anti- $\mathrm{g}_{3} \mathrm{t}$, anti-g $\mathrm{g}_{4}, \mathrm{~g}_{5}, \mathrm{p}$ and anti-f 
sera, which react with single factors $\mathrm{g}_{3}, \mathrm{~g}_{4}$ and $\mathrm{f}$, respectively, were used as the selective agents. An overnight broth culture of TR I 6 was streaked in line on plates of semisolid medium each containing one of the anti-factor(s) sera at a concentration at which the growth of the inoculated organisms spread very slowly. After incubation for $48 \mathrm{~h}$, several faster-spreading swarms were detected on every plate. The average number of swarms per streak (about $\mathrm{I} \times 10^{8}$ bacteria were inoculated per streak) was two in plates containing anti- $\mathrm{g}_{3} \mathrm{t}$ serum, one in those containing anti- $\mathrm{g}_{4}, \mathrm{~g}_{5}, \mathrm{p}$ serum, and eight in those containing anti-f serum. Five such mutant clones were isolated from each of the three types of selective plates. Their strain numbers are listed in Table 3. In slide agglutination tests, all of the mutants were still weakly agglutinated by the anti-factor(s) sera used for their selection. A faster spreading clone, SJ I 565 , was isolated from $\mathrm{SJ}$ 1 556 , a mutant selected with anti-f serum, on a plate containing anti-f serum at sufficient concentration to immobilize su 1556 cells.

Motility of these mutants in broth was normal judged by microscopic observation. Their spreading ability in semisolid medium without antiserum was not significantly different from that of the parental organisms. Flagellar antigens of these mutants will be described fgM I to fgM I6 (Table 3) in accordance with the description of the mutant $\mathrm{i}$ antigens by Joys \& Stocker (1966).

\section{Table 3. Agglutination of the antigen mutants and the wild-type strain by anti-fg (wild-type) and anti-factor(s) sera}

Titres were measured by tube agglutination and are expressed as the reciprocal of the highest dilution showing activity.

\begin{tabular}{|c|c|c|c|c|c|c|}
\hline \multirow[b]{2}{*}{$\begin{array}{l}\text { Strain } \\
\text { no. }\end{array}$} & \multirow[b]{2}{*}{$\begin{array}{c}\text { Flagellar } \\
\text { antigen }\end{array}$} & \multirow{2}{*}{$\begin{array}{l}\text { Anti-factor(s) } \\
\text { serum used for } \\
\text { selection }\end{array}$} & \multicolumn{4}{|c|}{ Agglutination titre of antiserum } \\
\hline & & & $\begin{array}{c}\text { Anti-fg } \\
\text { (wild-type) }\end{array}$ & Anti-g $g_{3}, t$ & Anti-g $g_{4}, g_{5}, p$ & Anti-f \\
\hline TR I 6 & fg (wild-t) & pe & 12,800 & 400 & 400 & 800 \\
\hline SJ I 575 & fgM I & & 12,800 & 40 & 400 & 800 \\
\hline sJ 1576 & $\mathrm{fgM}_{2}$ & & 3,200 & 40 & 80 & 80 \\
\hline SJ 1577 & $\mathrm{fgM}_{3}$ & anti-g $g_{3}, t$ & 6,400 & 40 & 120 & 160 \\
\hline SJ I 578 & $\mathrm{fgM}_{4}$ & & 3,200 & 40 & 80 & 80 \\
\hline SJ I579 & fgM 5 & & 6,400 & 40 & 120 & 160 \\
\hline SJ I 570 & fgM 6 & & 3,200 & 80 & 80 & 80 \\
\hline SJ 1571 & $\mathrm{fgM}_{7}$ & & 6,400 & 400 & 120 & 800 \\
\hline SJ 1572 & fgM 8 & anti-g $g_{4}, g_{5}, p$ & 3,200 & 80 & 80 & 120 \\
\hline SJ I 573 & fgM9 & & 6,400 & 400 & 40 & 800 \\
\hline SJ I 574 & fgM Io & & 6,400 & 400 & 20 & 800 \\
\hline SJ I 556 & fgM I I & & 12,800 & 400 & 400 & 400 \\
\hline SJ I 557 & fgM 12 & & 6,400 & 400 & 400 & 80 \\
\hline SJ $155^{8}$ & fgM I3 & & 6,400 & 400 & 400 & 80 \\
\hline SJ I 559 & fgM I4 & anti-f & 6,400 & 400 & 400 & 80 \\
\hline SJ 1560 & fgM I 5 & & 6,400 & 400 & 400 & 80 \\
\hline SJ I 565 & fgM I 6 & & 6,400 & 400 & 400 & 80 \\
\hline
\end{tabular}

Serological nature of the mutants

The mutants were first examined for their reactivity with anti-fg (wide-type) serum and the three anti-factor(s) sera (Table 3). Agglutination titre of the mutant antigens measured by anti-fg serum varied from the same to $I / 4$ of that for the homologous antigen. Reactivity of each mutant antigen to anti-factor(s) serum used for selection was decreased from $\mathrm{I} / 2$ to $\mathrm{I} / 20$ in titre compared to that of the wild-type antigen. Four mutants selected by anti- $\mathrm{g}_{3}, \mathrm{t}$ and two mutants selected by anti- $\mathrm{g}_{4}, \mathrm{~g}_{5}, \mathrm{p}$ sera, had 
been weakened in their reactivity to all three anti-factor(s) sera simultaneously, but no mutant had completely lost reactivity to any one of the anti-factor(s) sera. Antisera were then prepared against five representative mutant antigens, fgM 2, fgM 6, fgM I I, $\mathrm{fgM}_{12}$ and fgM 16 . Antisera having almost the same titre as anti-fg (wild-type) serum on the homologous antigens were obtained.

To examine whether the mutant antigens had lost some antigenic specificities present on the wild-type antigen and whether they had gained some specificities absent from it, cross-absorption-agglutination of antisera was then carried out between the wildtype antigen and each of the five mutant antigens. Antisera were repeatedly absorbed with absorbing organisms as described in Methods, and tested for their remaining activity after every absorption. In each case, activity not only on absorbing antigen but also on homologous antigen was completely removed within six serial absorptions. In control experiments, in which anti-fg serum was repeatedly absorbed with a nonflagellate mutant strain derived from TR I6, the titre of the antiserum was not lowered after six serial absorptions.

Thus these mutant antigens had neither completely lost any specificity present on the wild-type antigen nor gained any specificity absent from it and that one or more antigenic determinants had been slightly altered while retaining weak cross-reactivity with the original ones.

(a) TR 16

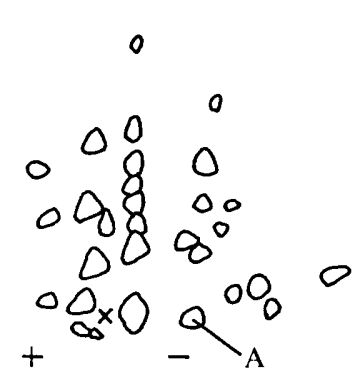

(b) Ss 1557 (fgM 12)<smiles></smiles>

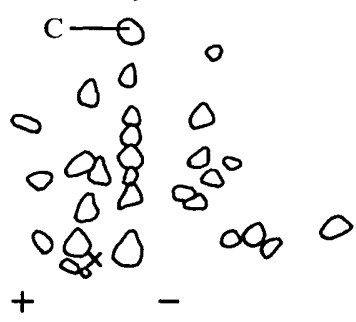

(c) SJ 1570 (fgM6)

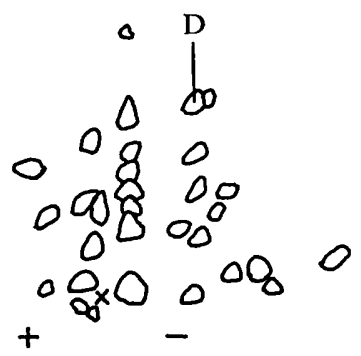

Fig. I. Tracing of the tryptic peptide maps of flagellins of TR I6 and its antigen-type mutants, SJ I 557 (flagellar antigen:fgM I 2) and sJ 1570 (fgM6). Electrophoresis at pH 6.7 followed by descending chromatography with $n$-butyl alcohol + acetic acid + water $(3+\mathrm{I}+\mathrm{I}$, by vol.).

\section{Transductional analysis of the mutants}

To determine the sites of mutation transduction of $H_{I}$ was carried out from the representative mutants to SJ 24I, a phase-I stable strain of Salmonella abortus-equi, whose $H_{I}$-allele is $H_{I-a}$. Mutants SJ 575 (phase-I antigen: fgM I), SJ I576 (fgM 2), SJ I570 (fgM6), SJ I57I (fgM 7), SJ I556 (fgM II) and sJ I557 (fgM I 2) were used as donors. The transduction mixture was streaked in line on semisolid medium containing a concentration of anti-a serum sufficient to inhibit the spreading growth of the recipient. The developing swarms, i.e. transductional clones, which received the $\mathrm{HI}_{\mathrm{I}}$ allele from the donor in place of the $H_{I}-a$ of the recipient, were isolated on nutrient agar plates. After overnight incubation, they were then spotted on three different semisolid media containing anti- $\mathrm{g}_{3}, \mathrm{t}$ serum (plate-I), anti- $\mathrm{g}_{4}, \mathrm{~g}_{5}, \mathrm{p}$ serum (plate-2) and anti-f serum (plate-3), at concentrations of antisera just sufficient to immobilize the wild-type TR I6. In control experiments, in which the donor organisms only were 
spotted, SJ 1576 and SJ I 570 showed spreading growth on all three plates, while SJ I 575 could spread only on plate-I, SJ I 57 I on plate-2, and SJ I 556 and SJ I557 on plate-3. In each transduction 100 to I 50 transductant clones were examined for their spreading ability in this manner. All the transductional clones showed the same spreading pattern as their respective donor strains. Thus the flagellar antigens of all the transductional clones are the same as those of the donor mutants, indicating that the sites of mutation in these mutants are inseparable from the $H_{I}$ gene.

\section{Peptide patterns of the mutant antigens}

The tryptic peptide maps of flagellins from sJ I 557(fgM I 2) and sJ I 570(fgM 6) were compared with that of the wild-type flagellin (Fig. I). About 30 peptide spots were seen on every peptide map. On the map for SJ 1557 flagellin two new spots, B and C in Fig. $\mathrm{I} b$, absent from the wild-type flagellin were seen, while spot $\mathrm{A}$ in Fig. I $a$, disappeared. On the map for SJ 1570 flagellin a spot, D in Fig. I $c$, absent from the wild-type flagellin was seen, but no other difference was detected.

\section{DISCUSSION}

Three mechanisms have been shown to be responsible for the genetic alteration of flagellar antigens in Salmonella: (I) phase variation (Andrews, I922); (2) structural modification of flagellin by gene $\mathrm{nml}$ (Stocker, McDonough \& Ambler, I96I); (3) changes in the primary structure of flagellin resulting from a mutation in $H$ genes. Mutants with an altered form of antigen i isolated by Joys \& Stocker (I966) belong to the third class. Transductional and finger-printing analyses showed that the mutants described above also belong to this class. Serological analysis has shown here that the specificity of a factor or factors in the mutant antigens had been slightly altered while retaining weak cross-reactivity with the wild-type antigen, and no specificities had been completely lost and no new specificities appeared. In this respect, these mutants differ from the mutant $i$ antigens isolated by Joys \& Stocker (I966) which had lost some of the antigenic specificities of the wild-type antigen and had gained some new specificities. The use of anti-factor(s) sera for selection in the present experiments probably permitted the detection of mutants with only slightly altered antigens. This difference between the mutants of Joys \& Stocker and those isolated here may not be absolute but represent parts of a series. SJ I 565 (phase-I antigen:fgM I6) was obtained from SJ I 556 ( $(\mathrm{gM}$ I I) on a plate containing anti-f serum at sufficient concentration to immobilize sJ $\mathrm{I} 556$. The agglutination titre of antigen $f g M$ I 6 against anti-f serum was much more reduced than that of antigen fgM I I. By further increasing the concentration of anti-f serum in the selection medium mutants completely lacking the factor $f$ may be obtained. Such different degrees of antigenic alteration resulting from mutational changes in $H$ genes reflect the variety of the conformational changes in the flagellin molecule, primarily determined by the site or sites of amino acid substitution and the amino acids involved.

Mutant antigens were classified into two groups; those in which only a single factor had altered and those in which all three factors examined had simultaneously altered. Antigens of all the mutants selected with anti-f antibody belong to the former group, while antigens of four mutants selected with anti- $g_{3}$ antibody and two mutants selected with anti- $\mathrm{g}_{4}$ antibody belong to the latter. The specificity of an antigenic determinant 
is altered not only by a change in the primary structure of the determinant itself but also by a change in primary structure of some other part of the molecule (Yamaguchi \& Iino, 1969). Mutant antigen fgM6 was altered in three factors simultaneously but only a single change in its tryptic peptide map was detected. Since the antigenic specificity-determining regions for factors $\mathrm{g}_{3}, \mathrm{~g}_{4}$ and $\mathrm{f}$ are known to be distributed separately in the $H_{I}$ gene (Yamaguchi \& Iino, I969), it is concluded that in mutant antigen fgM 6 alteration of some or all of the three factors resulted not from a simultaneous change in the primary structure of the antigenic determinants corresponding to the altered factors but through a conformational change accompanying a mutational change at a separate site of the molecule.

The frequency of the occurrence of antigen mutants selected with anti-f antibody was four to eight times higher than that for mutants selected with anti- $\mathrm{g}_{3}$ or anti- $\mathrm{g}_{4}$ antibody. It is inferred, therefore, that the antigenic determinant $f$ is more susceptible to a conformational change than determinants $\mathrm{g}_{3}$ and $\mathrm{g}_{4}$.

On the tryptic peptide map of SJ 1557 (fgM I2) flagellin two new spots appeared while spot $\mathrm{A}$ of the wild-type flagellin disappeared. This suggests that an amino acid residue in peptide $A$ had been substituted by arginine or lysine in the mutant flagellin. Although only the factor $\mathrm{f}$ had been altered in this mutant antigen, it is not yet certain whether peptide A contains the determinant $\mathrm{f}$ or is a part of it.

Motility observed both microscopically and by spreading ability in semisolid medium was normal in these antigen mutants. Several kinds of flagella-shape mutants associated with an alteration in cellular motility have been isolated from Salmonella strains and shown to carry mutations in genes $\mathrm{HI}_{\mathrm{I}}$ or $\mathrm{H}_{2}$ (Iino, 1962; Iino \& Mitani, 1967). The flagellin in these mutants is also assumed to have an altered conformation. However, no differences in antigenic specificity between the flagella of these mutants and their parents has been detected. These facts suggest that antigenic determinants and the region determining the shape of flagella are located apart from each other in the flagellin molecule so that they can change independently.

\section{REFERENCES}

ANDREws, F. W. (1922). Studies in group agglutination. I. The Salmonella group and its antigenic structure. Journal of Pathological Bacteriology 25, 51 5-521.

AsakuRA, S., Eguchi, G. \& InNo, T. (1964). The reconstitution of bacterial flagella in vitro. Journal of Molecular Biology 10, 42-56.

IINo, T. (1962). Curly flagellar mutants in Salmonella. Journal of General Microbiology 27, 197-I 75.

IrNo, T. (1969). Genetics and chemistry of bacterial flagella. Bacteriological Reviews 33, 454-475.

IINo, T. \& MitANI, M. (1967). A mutant of Salmonella possessing straight flagella. Journal of General Microbiology 49, 8I-88.

Joys, T. M. \& STOCKER, B. A. D. (1966). Isolation and serological analysis of mutant forms of flagellar antigen $i$ of Salmonella typhimurium. Journal of General Microbiology 44, 12I-I 38.

JoYs, T. M. \& STOCKER, B. A. D. (I969). Recombination in $H r$, the gene determining the flagellar antigen-i of Salmonella typhimurium; mapping of $\mathrm{HI}$ and fa mutations. Journal of General Microbiology 58, 267-275.

Kauffmann, F. (1964). Das Kauffman-White Schema. In World Problem of Salmonellosis, pp. 21-66. The Hague: Dr W. Junk-Publishers.

Kerridge, D., Horne, R. W. \& Glauert, A. M. (I962). Structural components of flagella from Salmonella typhimurium. Journal of Molecular Biology 4, 227-238.

McDonough, M. W. (I962). Tryptic peptide maps of mutant Salmonella flagellins. Biochemical Journal 84, 114. 
McDonough, M. W. (1965). Amino acid composition of antigenically distinct Salmonella flagellar protein. Journal of Molecular Biology 12, 342-355.

Stocker, B. A. D., MCDonough, M. W. \& AMBLeR, R. P. (196I). A gene determining presence or absence of $\epsilon-N$-methyllysine in Salmonella flagellar protein. Nature, London 189, 556-558.

YAMAGUCHI, S. \& IINO, T. (1969). Genetic determination of the antigenic specificity of flagellar protein in Salmonella. Journal of General Microbiology 55, 59-74. 